\title{
Mobile Target Tracking Using a Reconfigurable Low Earth Orbit Constellation
}

\author{
Sarah J. Morgan ${ }^{a *}$, Ciara N. McGrath ${ }^{b, \dagger}$, and Olivier L. de Weck ${ }^{a, *}$ \\ ${ }^{a}$ Department of Aeronautics and Astronautics, Massachusetts Institute of Technology, Cambridge, MA 02139, USA \\ ${ }^{b}$ University of Strathclyde, Glasgow, Scotland G1 1XJ, United Kingdom
}

\begin{abstract}
Traditional Earth-observing satellite constellations must preselect orbits to match established mission objectives, inherently limiting the capability of the system to respond to dynamic events. A more flexible approach to constellation design which maneuvers satellites to new orbits throughout the mission lifetime can enable the tracking of mobile targets; such a concept will be explored and evaluated in the following paper. Currently, an existing approach for creating a reconfigurable constellation of satellites (ReCon) demonstrates some increased functionality. However, the repeating ground track (RGT) orbits used are only well-suited for static ground targets (i.e. particular latitude and longitude points) as they are defined by their repetitious passes over the particular points on the globe; inherently these orbits are not ideal for tracking mobile targets such as ships, oil spills, hurricanes, and other weather events. By addressing this gap and designing the constellation to follow these mobile targets, data return can be of higher quality and quantity leading toward improved predictions of future movements. Traditional non-maneuverable satellites may of course capture mobile feature data; however, the quality and quantity of data returned is driven by the satellites' orbits which were not designed for the particular mobile target. A proposed concept of operations that models possible sequential satellite maneuvers as a graph has shown promise for tracking mobile targets in the case of a single satellite. In the following paper, this concept is further explored by the addition of an optimizer to this method, applied to both a single satellite and a constellation of maneuverable satellites, like ReCon. Results for a tropical storm case study indicate that with relatively low amounts of delta-V per satellite, many possible maneuver combinations are found which result in improved accesses with greater total access time and closer passes. Trade-offs are shown between total target access time, mean distance to targets upon access, and total delta-V used. The performance of increased constellation size is also explored. By having satellites dynamically respond to mobile targets such as hurricanes, satellite imagery and remote sensing can be of better quality and even contribute to better predictions for developing storms.
\end{abstract}

\section{Introduction}

$\mathrm{T}$ YPICAL Earth-observing satellite missions utilize static orbits, which usually do not change throughout the mission lifetime. An approach which adds reconfigurability to these missions can allow the satellites to respond dynamically to events on the Earth by enabling satellites to modify their orbits as mission focus shifts [1]. One concept of a reconfigurable constellation discussed by Legge and Miller includes a nominal Global Observation Mode (GOM) in which satellites are positioned in a constellation to provide even, global coverage. If a localized event of interest occurs, a subset of satellites can then be reconfigured to a Regional Observation Mode (ROM) utilizing repeating ground track (RGT) orbits to obtain better coverage and improved revisit characteristics over the specific latitude and longitude point of the event [1]. However, in the case of particular remote sensing missions such as storm monitoring, oil spills, wildfires, or animal migrations, this specific latitude and longitude point may change over time. There is an opportunity to add to the flexible architecture of the reconfigurable constellation concept by further maneuvering satellites to dynamically respond to mobile targets. This can likewise address an existing gap in the remote sensing of tropical storms.

Satellite data currently used for hurricane forecasting draws from two main sources: monolithic GEO satellites viewing large swaths of the Earth constantly at low-medium spatial resolution and polar-orbiting LEO satellites providing higher spatial resolution observations but with varying temporal resolution [2]. Between these two data

\footnotetext{
*Graduate Student, Department of Aeronautics and Astronautics.

${ }^{\dagger}$ Research Associate, Department of Electronic and Electrical Engineering.

$\stackrel{+}{+}$ Professor, Department of Aeronautics and Astronautics.
} 
sources there is large contextual awareness of the tropics provided by the GEO satellite observations, along with more temporally-scattered but localized information from the polar-orbiting satellites; however there exists an opportunity for a more dynamic response to possible storms. An alternative approach of utilizing a reconfigurable constellation, as described above, can augment the sporadic temporal sampling of polar-orbiting satellites. This method can help improve hurricane intensity forecasting by allowing more high-resolution accesses of the storm (especially in the early stages of tropical storm formation) [3]. This technique can also allow for targeting specific regions of the storm for data collection, like the eye, which frequently is valuable for hurricane intensity forecasting [3].

Prior work done by McGrath et al. shows a potential application of this method, allowing a single satellite to maneuver to provide multiple targeted observations of a tropical storm as it moves across the ocean. This work, extensions of it, and the application of this method to a constellation will be discussed below.

\section{Prior Work}

McGrath et al. demonstrates a method of planning sequential low-thrust maneuvers to provide improved quality of flyovers of a hurricane when compared to a non-maneuvering satellite. Specifically, this method involves marking a series of latitude/longitude way-points along the hurricane track to be reached in an associated time window. These way-points and their associated time window will henceforth be called targets.

The method laid out by McGrath et al. involves calculating all possible maneuver options, bounded by a maximum delta-V and duration, and modeling these options as a graph. Each node in the graph represents the state of the satellite at a flyover of the target and each edge describes the maneuver required to achieve that state. The preferred set of maneuvers can then be selected from the graph [4]. For example, the shortest path in terms of lowest delta-V can be found by weighting the edges according to the delta- $\mathrm{V}$ used for the maneuver and applying a shortest path algorithm, such as Dijkstra's algorithm [5]. To generate a large number of options relatively quickly, an analytical method of calculating low-thrust maneuvers which either raise or lower orbital altitude is used [4] [6]. It should be noted that the application of the graph method inherently involves discretely exploring the design space. For example, possible delta-V options are explored in $0.5 \mathrm{~m} / \mathrm{s}$ increments.

The results from the paper by McGrath et al. show promise. For a case study of Typhoon Megi, a powerful storm in the Pacific in 2010, with five planned flyovers at 2.5-day intervals, a non-maneuvering satellite with a 200km field of view (FOV) achieves only one flyover that has the eye of the storm in view. Of the remaining four intended flyovers of targets, only three are achieved in the associated time window. Two possible maneuvering solutions are identified. One solution describes accessing four targets in their associated time window utilizing $13.5 \mathrm{~m} / \mathrm{s}$ delta- $\mathrm{V}$, with the eye fully in view in three of these flyovers. Another solution has the eye fully in view for all five target flyovers utilizing $20.5 \mathrm{~m} / \mathrm{s}$ delta-V, with one of the targets being accessed outside of the associated time window. [4]

\section{Method}

The extension of prior work done by McGrath et al. is two-fold. First, an optimizer will be used to select possible maneuvers rather than finding these through a graph search. Secondly, the use of multiple satellites, both 2- and 3 -satellite cases, will also be explored. This will allow for further viewing opportunities of the targets and allow for more maneuver opportunities. The design space will again be explored utilizing an optimizer for more efficient investigation.

It was noted through initial work to validate results shown in [4] that the design space of low-thrust maneuvers can be highly sensitive to discretization, and that better solutions may exist in between intervals. While the graph method allows for a sampling of the design space, a continuous exploration allows for the possibility of finding niche solutions which may have better performance characteristics. Therefore, exploring the space of possible sequential maneuvers in a continuous way may be reveal other solutions. Additionally, it was noted in initial exploration that a greedy solution which selected the 'best' maneuver (e.g. closest pass or lowest delta-V) for each target typically had terrible performance overall, as this mobile target tracking problem is akin to a traveling salesman problem.

\section{A. Problem Setup}

To form a comparison to results shown by McGrath et al. the same general problem setup is used as in [4]. The characteristics of the satellites(s) and the initial orbit are shown in Table 1; small 3U CubeSats are used with electrospray thrusters [4]. When 2- or 3- satellite cases were evaluated, these were placed in the same orbits, only shifting the Right Ascension of Ascending Node (RAAN) by 20 degrees and 40 degrees respectively. 
Table 1 Satellite characteristics and orbit parameters. [4]

\begin{tabular}{cc}
\hline Characteristic & Value \\
\hline \hline Mass $(\mathrm{kg})$ & 4 \\
\hline Maximum Thrust $(\mathrm{mN})$ & 0.35 \\
\hline Altitude $(\mathrm{km})$ & 703 \\
\hline Inclination $(\mathrm{deg})$ & 40 \\
\hline RAAN at Epoch $(\mathrm{deg})$ & 0 \\
\hline Argument of Latitude at Epoch $(\mathrm{deg})$ & 0 \\
\hline
\end{tabular}

The same Typhoon Megi case study is used, as in [4] with targets located at the center of the eye of the storm, spaced out at 2.5 day intervals along the typhoon's track. A viewing window of 20 hours on either side of each target time was allowed. In the simulation, track data collected at 6 hour time intervals was used [7] and interpolation between these latitude and longitude points was performed to approximate the actual storm path.

Table 2 Typhoon Megi locations at 2.5 day intervals. [7]

\begin{tabular}{cccc}
\hline Date and Time & Days Since Epoch & Latitude (deg) & Longitude (deg) \\
\hline \hline 13 Oct 2010 00:00:00.000 UTCG & 2.5 & 11.9 & 141.4 \\
\hline 15 Oct 2010 12:00:00.000 UTCG & 5 & 15.7 & 135.5 \\
\hline 18 Oct 2010 00:00:00.000 UTCG & 7.5 & 17.5 & 123.3 \\
\hline 20 Oct 2010 12:00:00.000 UTCG & 10 & 18.4 & 117.2 \\
\hline 23 Oct 2010 00:00:00.000 UTCG & 12.5 & 23.4 & 118 \\
\hline
\end{tabular}

The overall procedure of the simulation is as follows: after being initialized at epoch, the satellite(s) enter the first maneuver period during which a low thrust maneuver of a particular delta- $\mathrm{V}$ is conducted. The duration of the maneuver is fixed such that the maneuver must be completed at the start of the first viewing window, limiting the total delta-V that can be generated during each maneuver period, with an absolute maximum of $15 \mathrm{~m} / \mathrm{s}$ per maneuver. These maneuvers are raising and lowering maneuvers computed as in [6]. The satellite(s) are then propagated with J2 effects through the viewing window, during which any accesses of the storm are recorded. An access of the storm is defined as whenever the eye is within the field of view (FOV) of the satellite. In other words, whenever the eye of the storm is within 100km of the sub-satellite point, according to the Haversine distance formula. The propagation steps through time in 10 second intervals. The total number of intervals during which an access of the storm occurs is recorded; this is used as an approximation for total target access time. At the end of the first viewing window, the second maneuver period begins, and this process is repeated for all five targets. This method differs slightly from [4] as this includes a fixed maneuver duration that ends at the start of each viewing window, however this only slightly reduces the achievable delta- $\mathrm{V}$ options. In summary, given the delta- $\mathrm{V}$ for each maneuver, the simulation will output all access of the storm during the target viewing windows, including the total access time, distance to the target at each access, and time of each access. If no access of the storm is possible during a given viewing window regardless of the maneuver attempted, the satellite will simply propagate during the maneuver period to conserve delta-V.

To further describe this procedure, a plot in Fig. 1 shows the storm's track in red, overlaid with flyovers from a sample single satellite solution as in Table 3. The FOV of the satellite at flyovers is shown in yellow. The delta-V maneuvers required to complete this are shown in the graph below, with the resulting semi-major axis change over the course of the simulation shown below this. This was generated in Systems Tool Kit (STK) from Analytical Graphics, Inc. 

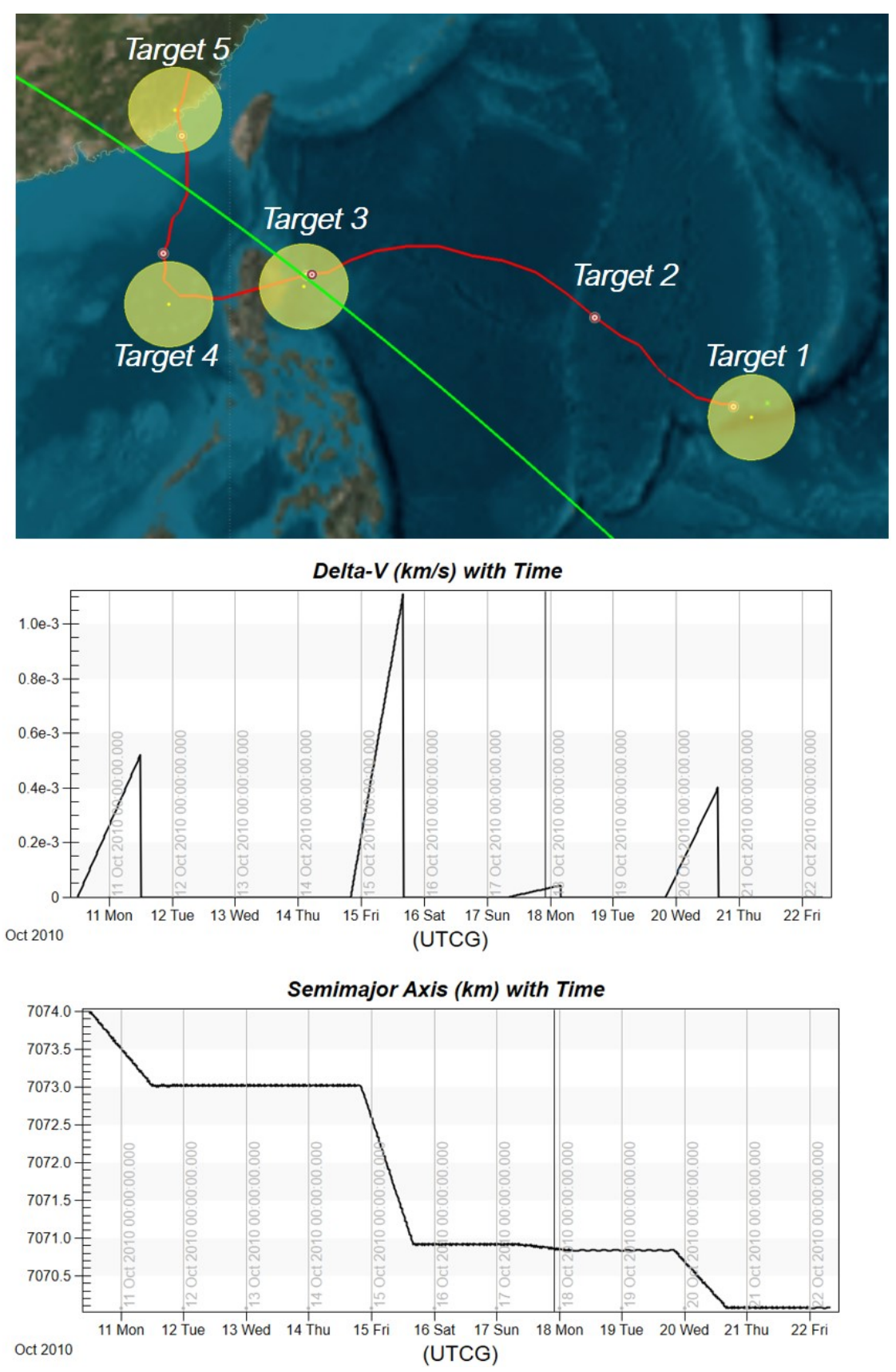

Fig. 1 Sample single satellite case modelled in STK.

\section{B. Optimization}

As mentioned previously, an optimizer was used to select possible maneuvers rather than enumerating discrete possibilities with the graph method. The optimizer was allowed to control the amount of delta-V used for each maneuver ( 5 total, as there were 5 targets) and was constrained by a maximum delta-V limited by possible maneuver duration. The optimization had the objectives to minimize the mean distance to each target each time an access occurred, minimize total delta-V used, and maximize the amount of time each target was in view. Note that no distinction was made between longer continual accesses and shorter frequent accesses, which should be further explored.

A genetic algorithm run through pymoo, specifically a BRKGA (Biased Random Key Genetic Algorithm), was used for this exploration, which utilized both elitism and random restarts [8]. A brief design of experiments of the algorithm 
parameters was conducted to obtain good convergence performance with this algorithm.

By computing these three objectives, more trade-offs may be apparent than through the graph search methodology. Additionally, it should be noted that these objectives are cumulative, representing the performance of the satellite(s) over the entire series of maneuvers, discouraging greedy maneuvers that may prohibit future accesses, as discussed in [4].

\section{Results}

The multi-objective optimization was carried out for a single satellite as well as 2- and 3- satellite constellations. Non-dominated solution sets for each of these cases are shown below. These solutions represent essentially the best performing solutions found by the optimizer - those which cannot improve in one objective without degrading in another. These plots demonstrate trade-offs in the solution space and sample solutions from these sets are shown to demonstrate the advantages of adding maneuverability and sample specific performance characteristics.

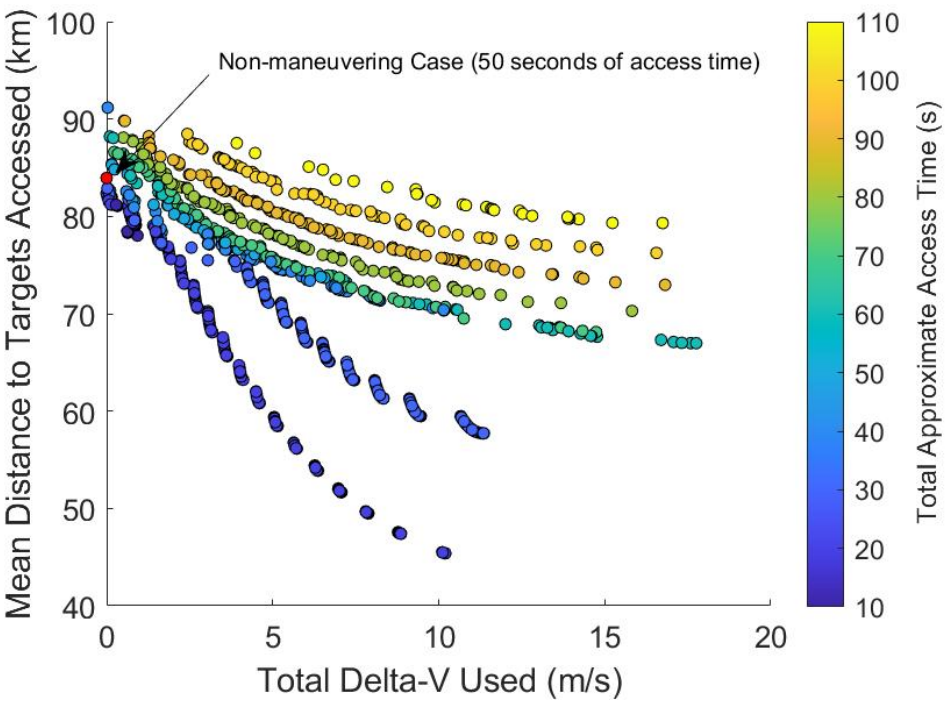

Fig. 2 Non-dominated set of solutions for single satellite case.

There are a multitude of possible solutions. The non-dominated set resulting from optimization is shown in the graph (Fig. 2) with the non-maneuvering solution labeled. Trade-offs can broadly be identified in this non-dominated set. It is clear that increased delta-V is required to view the targets from the same mean distance for the same number of accesses. Similarly, greater delta-V must be used to get closer to the targets for the same number of accesses; this trend is shown by the downward curving series of points across the plot. A decision maker evaluating possible options for this satellite may look at the trade-offs between greater total access time and closer passes for a fixed amount of delta-V.

A sample maneuvering solution is shown in Table 3 compared to the non-maneuvering case. This summarizes the accesses of each target, including the total access time over each target, the mean distance to the eye of the storm over all accesses for each target, delta- $\mathrm{V}$ used for each maneuver, and whether the eye is in view during any of the accesses. It should be noted that, as McGrath et al. mentioned, there is no maneuver combination that can enable a pass of the second target in the target time window within the delta- $\mathrm{V}$ restrictions in the single satellite case. Therefore, the satellite simply propagates through the maneuver period for the second target. Additionally, if one prioritized obtaining a full view of the eye of the storm, defined as a pass of closer than $80 \mathrm{~km}$ assuming the eye radius may be up to 20km [4], this would not be achievable for all targets in this single satellite case. To view the first target within $80 \mathrm{~km}$, the first maneuver must be a raising maneuver of at least $0.7 \mathrm{~m} / \mathrm{s}$. However, a large lowering maneuver is required to then obtain an equivalently close pass of the third target, which is not achievable within the constraints of the problem. Either greater thrust capability or maneuver duration would be required to view the eye of the storm in all 4 eligible targets. Therefore, the best possible performance of the single satellite maneuvering case includes viewing 4 targets, achieving at least one view of the eye at 3 of those targets. A sample solution requiring less than $2.5 \mathrm{~m} / \mathrm{s}$ delta-V is shown in Table 3 . 
Table 3 Sample comparison between non-maneuvering and maneuvering single satellites.

\begin{tabular}{|c|c|c|c|c|}
\hline \multirow[t]{2}{*}{ Target } & \multicolumn{4}{|c|}{ Non-maneuvering } \\
\hline & Delta-V (m/s) & $\begin{array}{l}\text { Total Access } \\
\text { Time (s) }\end{array}$ & $\begin{array}{l}\text { Mean Access } \\
\text { Distance }(\mathrm{km})\end{array}$ & Eye in View? \\
\hline 1 & - & 10 & 83.96 & No \\
\hline 2 & - & - & - & - \\
\hline 3 & - & 10 & 86.28 & No \\
\hline 4 & - & 10 & 83.15 & No \\
\hline 5 & - & 20 & 82.37 & Yes \\
\hline Summary & $0 \mathrm{~m} / \mathrm{s}$ & $50 \mathrm{~s}$ & $83.94 \mathrm{~km}$ & - \\
\hline \multirow[t]{2}{*}{ Target } & \multicolumn{4}{|c|}{ Maneuvering } \\
\hline & Delta-V (m/s) & $\begin{array}{l}\text { Total Access } \\
\text { Time (s) }\end{array}$ & $\begin{array}{l}\text { Mean Access } \\
\text { Distance }(\mathrm{km})\end{array}$ & Eye in View? \\
\hline 1 & 0.87 & 20 & 96.39 & No \\
\hline 2 & - & - & - & - \\
\hline 3 & 1.11 & 20 & 86.98 & Yes \\
\hline 4 & 0.04 & 30 & 88.45 & Yes \\
\hline 5 & 0.40 & 30 & 82.03 & Yes \\
\hline Summary & $2.43 \mathrm{~m} / \mathrm{s}$ & $100 \mathrm{~s}$ & $88.46 \mathrm{~km}$ & - \\
\hline
\end{tabular}

The same analysis was conducted for a two satellite case. The non-dominated set of solutions is shown in Fig. 3. Similar trade-offs to those shown in Fig. 2 exist, and it is clear that a multiple satellite case inherently has better performance, including naturally closer passes. Note that the $\mathrm{X}$-axis displays the average delta-V usage across the satellites rather than the total usage.

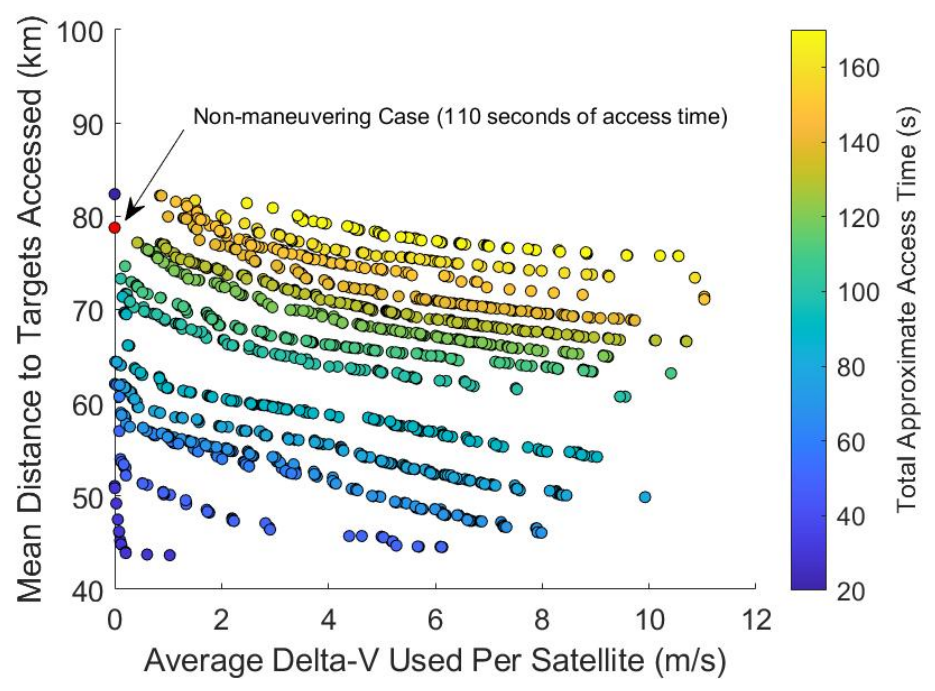

Fig. 3 Non-dominated set of solutions for 2-satellite constellation.

As mentioned for the single satellite case, a pass of the second target is still not achieved, and a pass within $80 \mathrm{~km}$ of the first target is still not possible in the constraints. Again, the best possible performance of this case includes viewing 4 targets, and achieving at least one view of the eye for 3 of those targets. However, the two satellite case shows heavily increased accesses of the third, fourth, and fifth target due to the addition of the second satellite. 
Table 4 Sample comparison between non-maneuvering and maneuvering constellation of 2 satellites.

\begin{tabular}{|l||l|l|l|l|}
\hline \multicolumn{1}{|l||}{ Target } & \multicolumn{5}{|c|}{ Non-maneuvering } \\
\cline { 2 - 5 } & Delta-V (m/s) & $\begin{array}{l}\text { Total Access } \\
\text { Time }(\mathrm{s})\end{array}$ & $\begin{array}{l}\text { Mean Access } \\
\text { Distance }(\mathrm{km})\end{array}$ & Eye in View? \\
\hline 1 & - & 10 & 83.96 & No \\
\hline 2 & - & - & - & - \\
\hline 3 & - & 10 & 86.28 & No \\
\hline 4 & - & 40 & 59.16 & Yes \\
\hline 5 & - & 50 & 76.75 & Yes \\
\hline \hline Summary & $0 \mathrm{~m} / \mathrm{s}$ & $110 \mathrm{~s}$ & $78.77 \mathrm{~km}$ & - \\
\hline \hline Target & \multicolumn{5}{|c|}{ Maneuvering } \\
\cline { 2 - 5 } & Total Delta-V & Total Access & $\begin{array}{l}\text { Mean Access } \\
\text { Distance }(\mathrm{km})\end{array}$ & Eye in View? \\
\hline 1 & $(\mathrm{~m} / \mathrm{s})$ & Time (s) & 96.48 & No \\
\hline 2 & 0.90 & 20 & - & - \\
\hline 3 & - & - & 89.23 & Yes \\
\hline 4 & 1.88 & 30 & 63.56 & Yes \\
\hline 5 & 1.92 & 60 & 76.34 & Yes \\
\hline \hline Summary & 0.23 & 60 & $81.40 \mathrm{~km}$ & - \\
\hline
\end{tabular}

Finally, the optimization was conducted with three satellites. As is clear in the figure, three satellites inherently perform better than the 1- or 2-satellite case, having a greater number of chances of accesses. Closer passes of the targets naturally occur, with greater total access time possible.

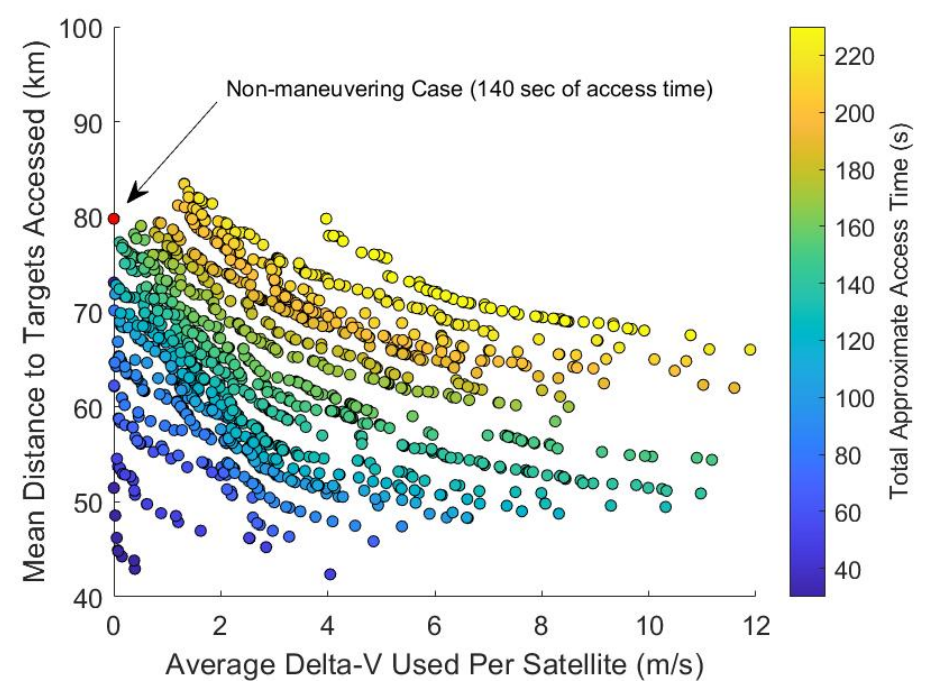

Fig. 4 Non-dominated set of solutions for 3-satellite constellation.

With the addition of the third satellite, all targets are in view in both maneuvering and non-maneuvering cases. The third satellite added (at 40 deg RAAN) achieves an access of the first target late in the viewing window, and achieves access of the second target as well. In the non-maneuvering case, all targets are in view, and only the fourth and fifth target are viewed within $80 \mathrm{~km}$. However, in the sample maneuvering case shown below, a view of the eye is possible at all 5 target locations within the desired time window. 
Table 5 Sample comparison between non-maneuvering and maneuvering constellation of 3 satellites.

\begin{tabular}{|c|c|c|c|c|}
\hline \multirow[t]{2}{*}{ Target } & \multicolumn{4}{|c|}{ Non-maneuvering } \\
\hline & Delta-V $(\mathrm{m} / \mathrm{s})$ & $\begin{array}{l}\text { Total Access } \\
\text { Time (s) }\end{array}$ & $\begin{array}{l}\text { Mean Access } \\
\text { Distance }(\mathrm{km})\end{array}$ & Eye in View? \\
\hline 1 & - & 30 & 85.55 & No \\
\hline 2 & - & 10 & 83.54 & No \\
\hline 3 & - & 10 & 86.28 & No \\
\hline 4 & - & 40 & 59.16 & Yes \\
\hline 5 & - & 50 & 76.75 & Yes \\
\hline Summary & $0 \mathrm{~m} / \mathrm{s}$ & $140 \mathrm{~s}$ & $79.96 \mathrm{~km}$ & - \\
\hline \multirow[t]{2}{*}{ Target } & \multicolumn{4}{|c|}{ Maneuvering } \\
\hline & $\begin{array}{l}\text { Total Delta-V } \\
(\mathrm{m} / \mathrm{s})\end{array}$ & $\begin{array}{l}\text { Total Access } \\
\text { Time (s) }\end{array}$ & $\begin{array}{l}\text { Mean Access } \\
\text { Distance }(\mathrm{km})\end{array}$ & Eye in View? \\
\hline 1 & 2.06 & 50 & 90.51 & Yes \\
\hline 2 & 0.24 & 30 & 91.23 & Yes \\
\hline 3 & 4.968 & 30 & 84.88 & Yes \\
\hline 4 & 4.1722 & 60 & 59.47 & Yes \\
\hline 5 & 0.466 & 60 & 71.88 & Yes \\
\hline Summary & $11.91 \mathrm{~m} / \mathrm{s}$ & $230 \mathrm{~s}$ & $79.79 \mathrm{~km}$ & - \\
\hline
\end{tabular}

This exploration confirms that for relatively low amounts of delta- $\mathrm{V}$ increased access time and closer passes of targets along a mobile target track are possible. In the case of a single satellite, less than $2.5 \mathrm{~m} / \mathrm{s} \mathrm{delta-V}$ doubled the total approximate access time. This analysis also has exposed distinct trade-offs in the design space of mobile target tracking through low thrust maneuvers; the trade between access time and distance to the target is one example. There likewise exists a trade-off between choosing increased maneuverability and increased numbers of satellites, as shown through the progression in solution space summarize in Fig 5. below. Legge and Miller has explored this in part. There is a sense of diminishing returns, as more coverage is naturally possible with increased number of satellites, and the advantage of maneuverability fades. Further investigation is warranted into the study of this in the case of mobile targets.
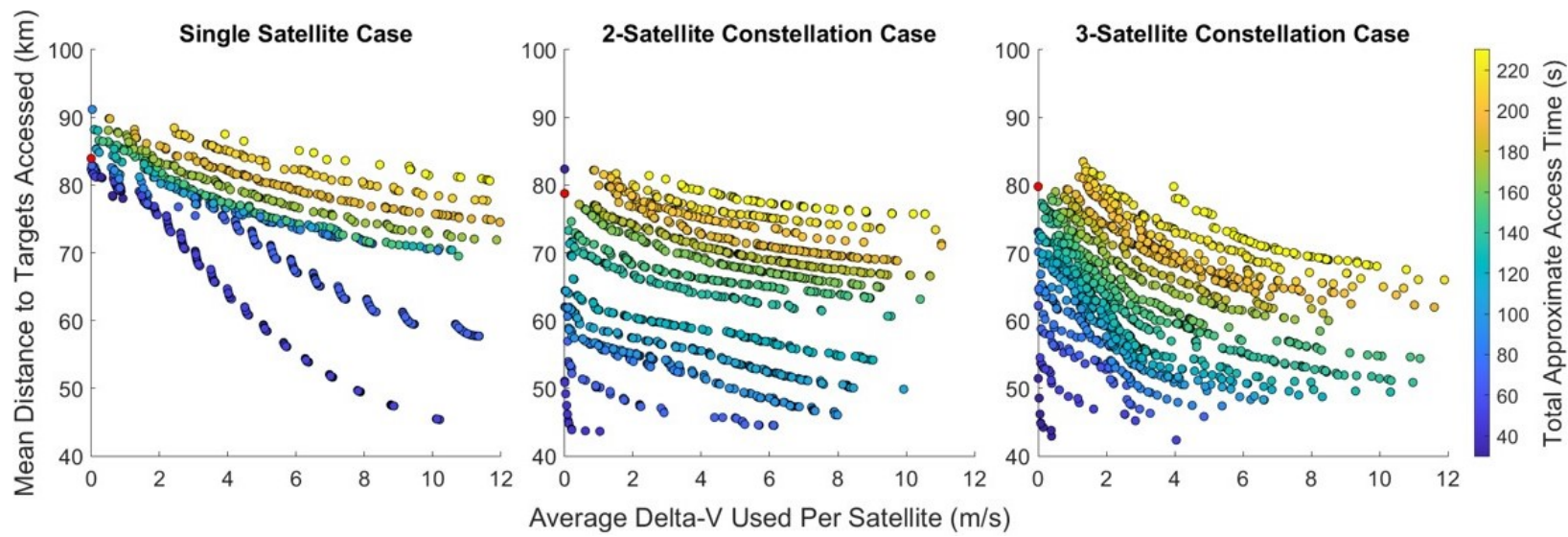

Fig. 5 Non-dominated sets of solutions for all evaluated cases. 


\section{Conclusion}

Overall, it is shown that for relatively low amounts of delta-V, satellites can be intelligently maneuvered to gain better quality and quantity of accesses of target way-points along a hurricane's track. The method presented by McGrath et al. can be enhanced with the addition of an optimizer, allowing for continuous design space exploration. The evaluation of the design space shown through this optimization method can be useful to decision makers; a greater number of accesses may be more vital in certain applications like early in a hurricane's lifetime [2] but a closer pass may be more vital for high resolution data-gathering.

In addition, it is shown that the same method of sequential low thrust maneuvers can be applied to a constellation with similar trade-offs. Further exploration of this space needs to be conducted to determine when an increased number of satellites in desirable orbits may meet the performance of several maneuverable satellites.

It is shown that this application of low-thrust maneuvers can allow for better quality and quantity of data gathered over a hurricane's track, which can lead to improved hurricane forecasting. The opportunities described in this paper for responsive satellite and constellation architectures also open the doors for other mobile target tracking applications. Additionally, given the reconfigurable constellation framework, this responsiveness allows for multiple missions to be conducted utilizing the same satellites, as the architecture allows for mission evolution with time.

\section{Acknowledgments}

S. Morgan thanks Dr. de Weck and Dr. McGrath for their advice and support throughout the writing of this paper. S. Morgan also thanks the Aerospace Corporation for the internship during which much of this preliminary work was developed.

\section{References}

[1] Legge, R. S., and Miller, D. W., “Optimization and valuation of recongurable satellite constellations under uncertainty,” Ph.D. thesis, Massachusetts Institute of Technology, 2014. URL http://dspace.mit.edu/handle/1721.1/7582

[2] McNally, T., Bonavita, M., and Thépaut, J.-N., "The Role of Satellite Data in the Forecasting of Hurricane Sandy," Monthly Weather Review, Vol. 142, No. 2, 2014, pp. 634-646. https://doi.org/10.1175/MWR-D-13-00170.1. URL https://doi.org/10.1175/MWRD-13-00170.1

[3] Ruf, C., Unwin, M., Dickinson, J., Rose, R., Rose, D., Vincent, M., and Lyons, A., "CYGNSS: Enabling the Future of Hurricane Prediction [Remote Sensing Satellites]," IEEE Geoscience and Remote Sensing Magazine, Vol. 1, No. 2, 2013 , pp. 52-67.

[4] McGrath, C., Clark, R., Werkmeister, A., and Macdonald, M., "Small satellite operations planning for agile disaster response using graph theoretical techniques," 2019. URL https://www.iac2019.org/ the paper was originally presented at the 70th International Astronautical Congress, 21-25 October 2019, Washington, D.C.; 70th International Astronautical Congress, IAC ; Conference date: 21-10-2019 Through 25-10-2019.

[5] Dijkstra, E. W., "A note on two problems in connexion with graphs," Numerische Mathematik, Vol. 1, No. 1, 1959 , pp. $269-271$. https://doi.org/10.1007/BF01386390. URL https://doi.org/10.1007/BF01386390

[6] Mcgrath, C., and Macdonald, M., "General Perturbation Method for Satellite Constellation Reconfiguration Using Low-Thrust Maneuvers," Journal of Guidance, Control, and Dynamics, Vol. 42, 2019, pp. 1-17. https://doi.org/10.2514/1.G003739

[7] Kitamoto, A., “Digital Typhoon: Typhoon 201013 (MEGI) - Detailed Track Information,” , Dec 2012. URL http://agora.ex.nii. ac.jp/digital-typhoon/summary/wnp/1/201013.html.en

[8] Blank, J., and Deb, K., “pymoo: Multi-objective Optimization in Python,” IEEE Access, 2020, pp. 1-1. 